\title{
СТРУКТУРНО-ЛЕКСЕМАТИЧЕСКОЕ СОДЕРЖАНИЕ ОЦЕНОЧНОЙ ДИКТЕМЫ В ДИАЛОГИЧЕСКОЙ РЕЧИ
}

\section{THE STRUCTURAL AND LEXEMIC CONTENT OF AN EVALUATIVE DICTEME IN DIALOGICAL SPEECH}

Yu. Medvedeva

Summary: The article is devoted to the description of an evaluative dicteme at the semantic, structural and lexematic levels. It provides the initial derivational structure of an evaluative sentence and discusses some typical deviations from the given normative structure occurring in dialogues. The results of the definitional analysis of the evaluative lexis are presented with special attention being paid to the dichotomy of extreme and non-extreme evaluation degrees capable of being implied in the semes of evaluative lexis and its subsequent dichotomous division.

Keywords: dialogical speech, evaluative dicteme, initial derivational structure, evaluative lexis, extreme evaluation.
Медведева Юлия Ивановна

Старший преподаватель, Финансовый университет при Правительстве Российской Федерации (Москва) Tea4english@yandex.ru

Аннотация: Статья посвящена описанию оценочной диктемы на семантическом, структурном и лексематическом уровнях. Дана деривационно-исходная структура оценочного предложения и описаны частотные варианты отклонений от данной нормативной структуры в построении реплик в диалогической речи. Представлены результаты дефиниционного анализа оценочной лексики в её дихотомическом делении на слова с семой предельной и непредельной степени оценки.

Ключевые слова: диалогическая речь, оценочная диктема, деривационноисходная структура, оценочная лексика, предельная оценка.

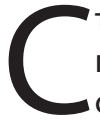
точки зрения уровневой структуры языка, как правило, каждая целая реплика диалога представляет собой диктему [1, с. 63]. Диктема является элементарной тематической единицей связной речи, включающей в себя либо одно предложение, «если только оно является полноценным, пропозиционно оформленным предложением (в том числе и однословным, т.е. структурно любым предложением), выражающим собственную тему («микротему»), а не случайным междометоподобным изглашением-выкриком» [1, с. 62], либо несколько предложений, объединённых выражаемой микротемой.

Семантическая структура оценочной диктемы включает в себя четыре базовых элемента: оценивающий субъект, оценочный предикат, объект оценки и основание оценки (последнее зачастую выражено имплицитно). Если диктема состоит из одного предложения, и это предложение обладает оценочной семантикой, то следует говорить об оценочной диктеме. В случае если диктема представляет собой несколько предложений, то диктема может считаться оценочной, если объединяющей эти предложения темой является выражение оценки, даже если не все эти предложения являются семантически оценочными. И наоборот, если в диктеме присутствуют оценочные денотемы или пропоземы, однако выражение оценки не является темой, объединяющей предложения диктемы, то она не может считаться оценочной.

Оценочной диктеме, как правило, свойственна оце- ночная ориентация, т.е. в случае если диктема представлена несколькими предложениями, зависимые предложения диктемы согласуются по оценочному знаку с ведущим предложением, одним или несколькими, образующими смысловой центр диктемы. Кроме того, зависимые предложения, как правило, поддерживают, раскрывают или усиливают оценку, заключённую в смысловом центре диктемы. Приведём пример:

Jimmy: God, how I hate Sundays! It's always so depressing, always the same. We never seem to get any further, do we? Always the same ritual. Reading the papers, drinking tea, ironing. A few more hours, and another week gone. Our youth is slipping away. Do you know that? [15, p. 40]

В приведённом примере первые два предложения представляют собой ведущие предложения, задающие оценочную ориентацию диктемы, а последующие раскрывают и дополняют выражаемую оценку. При этом зависимые предложения (Always the same ritual. Reading the papers, drinking tea, ironing. A few more hours, and another week gone.) фактически не обладают оценочной семантикой при их рассмотрении в изоляции, однако, являясь элементами оценочной диктемы, они приобретают оценочное значение. Из этого вытекает важность учитывания в диалогической речи оценочной ориентации диктемы.

С точки зрения синтаксической структуры, «простейшая деривационно-исходная структура оценочного 
предложения представляет собой такую предикативную конструкцию, в которой объект оценки выражается субъектом предложения, субъектом оценки выступает говорящий, а оценочный предикат состоит из связки и предикатива-оценки. При этом предикативом может выступать как оценочное прилагательное, так и качественно-оценочное существительное» [6, с. 111].

Таким образом, исходная структура может быть представлена формулами: $\mathrm{S}+\mathrm{P}\left(\mathrm{be}+\mathrm{Adj}_{\mathrm{ev}}{ }^{1}\right)$ и $\mathrm{S}+\mathrm{P}\left(\mathrm{be}+\mathrm{N}_{\mathrm{ev}}\right)$, которые актуализируются оценочными предложениями типа: "It was lovely, Monica" [10, p. 128], "They say the women are dusky and beautiful, and all the men are studs" [11, p. 145].

Данная простейшая предикативная конструкция служит «исходной базой для остальной синтактико-парадигматической системы оценочных предложений» [3, с. 40]. Так, оценочные существительные могут быть употреблены с дополняющими их значение и зачастую интенсифицирующими степень оценки оценочными прилагательными, как в следующем примере: "You're a low spy, an impudent, bare-faced liar, a common kitchen-cat who wriggles into the best rooms, gets herself fondled, and then spits" [16, p. 163].

К группе оценочной лексики относятся также глаголы, способные за счёт отрицательной или положительной оценочной коннотации выражать оценку действия. Примерами могут служить глаголы sneak, cheat, flatter, stink, degrade, fail, lose, show off, grovel, crawl, poison, gnaw, gloat, snare, smother, snarl, sicken, spit, maim, disfigure, murder, wreck, settle, succeed, win и др. Структурно-лексематическое содержание оценочного высказывания в таком случае будет отображено формулой $\mathrm{S}+\mathrm{P}\left(\mathrm{V}_{\mathrm{ev}}\right)$, где $\mathrm{S}$ - это актант, а $\mathrm{P}\left(\mathrm{V}_{\mathrm{ev}}\right)$ - оценочный предикат. Справедливым будет отметить, что в таком предложении, как правило, объектом оценки выступает не только действие, но и его актант. В примере "Oh, you're not going to start up that old pipe again, are you? It stinks the place out" [15, p. 4142] говорящий выступает против самой трубки из-за запаха, который она производит.

Согласно М.Н. Эпштейн, оценочная лексика делится на два класса. К первому классу относятся слова, значения которых содержат оценку, однако не указывается, к чему именно, к какому предмету или явлению относится эта оценка (good, bad, magnificent, wonderful). Эти слова «сугубо оценочные», их предметная соотнесённость выясняется из контекста.

Ко второму классу принадлежат «предметно-оценочные» слова, которые не только называют явление, но и сообщают оценку этого явления, то есть в словарной дефиниции данного типа слов обычно присутствуют как предметные, так и оценочные компоненты, причём последние могут записываться либо в виде словарной пометы, либо в виде оценочных слов, входящих в дефиницию [7, с. 19]. Например: vivacious - someone, especially a woman, who is vivacious has a lot of energy and a happy attractive manner - used to show approval [13].

Слова этого класса являются прагмемами, поскольку в их лексическом значении семантический аспект - отношение слова к обозначаемому явлению - неразрывно связан с прагматическим аспектом - отношением говорящего к предмету сообщения. Прагмемы более автономны, нежели слова первого класса, в коммуникативном плане - они вобрали контекст, или прагматическую ситуацию в ядро своего лексического содержания, в силу чего могут употребляться как законченные суждения о том, что они сами обозначают [7, с. 20].

Таким образом, употребление прагмем в оценочной диктеме позволяет выразить как оценочное, так и дескриптивное значение, выступающее имплицированным основанием оценки. В диктемах, в которых оценка выражена «сугубо оценочными» словами, превалирует собственно оценочное значение, при этом основание оценки выявляется собеседником как исходя из его картины мира, так и за счёт контекста.

Известно, что оценке свойственна градация, ввиду чего в пласте оценочной лексики выделяется дихотомия «предельно-» и «непредельно-оценочной» лексики, т.е. слов с семой предельной либо непредельной степени оценки. Арсенал средств, воплощающих предельный элемент оценочной шкалы, представлен прилагательными, производными от них наречиями и, в меньшей степени, существительными.

Группа предельно-оценочных прилагательных располагает к упорядочению: семы внутри группы пересекаются, «значение одного прилагательного зачастую передаётся через значение другого. Так, значения <...> прилагательных, выражающих мелиоративную предельную оценку, наиболее часто передаются через номинации excellent, extremely good, extremely enjoyable» [3, c. 39]. В ходе исследования были выделены следующие примеры: amazing, sensational, first-class, ideal, perfect, brilliant, marvellous, gorgeous, splendid, terrific, great, magnificent, glorious, stunning, heavenly, blissful, awesome, admirable, exquisite, outstanding. Значения прилагательных, выражающих пейоративную предельную оценку, наиболее регулярно передаются через номинации extremely bad, extremely unpleasant. Примерами могут выступать hellish, awful, dreadful, terrible, horrible, wretched, disgusting, vile, revolting, detestable, ghastly, appalling, gross, horrendous, monstrous, outrageous, devilish, abominable, atrocious, con-

1 Ev. - evaluation / оценка 
temptible, obscene, despicable.

При выделении группы предельно-оценочных существительных из общего пласта оценочной лексики учитывалось наличие в словарной дефиниции предельно-оценочных слов, типа perfect, ideal, supreme, extremely pleasant, extremely good, outstanding, exceptional, напримep, masterpiece - an extremely good example of something [14]. Были выделены такие существительные, как perfection, ideal, heaven, paradise, god, hero, bliss, masterpiece, horror, hell, nightmare, disaster.

Хотя часть выделенных существительных (наприmep, hero - a man who is admired for doing something extremely brave [13], god - a man who is extremely attractive [14]) обладают предметным значением, дефиниционный анализ показывает, что подавляющее большинство предельно-оценочных слов являются «сугубо оценочными», т.е. не обладают предметным компонентом значения. Представляется, что объяснением этому служит их внутренняя экспрессивность, то есть группа предельно-оценочных лексем используется прежде всего для интенсифицированного выражения эмоциональнооценочного отношения говорящего к объекту оценки. Именно в силу своей внутренней экспрессивности предельно-оценочные лексемы, обладающие предметным значением, зачастую употребляются метафорически, таким образом утрачивая его.

Лексемы с коннотативным оценочным значением всегда имплицируют непредельную степень оценки и могут выражать предельную оценку только при наличии специальных усилительных языковых средств, таких как наречия-интенсификаторы extremely, absolutely, completely и др.; наречия, являющиеся производными предельно-оценочных прилагательных; префиксы super-, extra-, hyper-, -ultra, выступающие имплицитными интенсификаторами оценочной семы слова.

В пласте предельно-оценочной лексики отсутствуют оценочные глаголы, которым, как правило, свойственна оценочная коннотация, вследствие чего для выражения предельной оценки действия употребляются предельно-оценочные наречия, присоединяемые к глаголу: "She's behaving admirably" [16, p. 35]; "Oh, I've been treating him shockingly!" [lbid: 42].

Наличие характерного выразительного арсенала предельной оценки, который располагает к упорядочению, делает возможным описание структур предельно-оценочных высказываний как $\mathrm{S}+\mathrm{P}\left(\mathrm{be}+\mathrm{Adj}_{\mathrm{ex.ev}}{ }^{2}\right), \mathrm{S}+$ $P\left(\right.$ be $\left.+N_{\text {ex.ev }}\right), S+P\left(b e+A d v_{\text {ex.ev }}+A d j\right), S+P\left(A d v_{\text {ex.ev }}+V\right)$, которые актуализируются оценочными предложениями типа: "I think this is - hateful!" [17, p. 86]; "The system's hell"
[18, p. 145]; "You've captained us magnificently" [8, p. 37].

Известно, что степень развернутости реплик в диалогической речи значительно варьируется, и их информативная полнота обеспечивается ситуативной обусловленностью речи и общностью апперцепционной базы говорящих. Понятие общности апперцепционной базы пересекается с понятием пресуппозиции (в одном из значений этого термина) и понимается как фонд общих знаний говорящего и слушающего, необходимый для понимания речи и включающий в себя как общие знания людей об окружающем мире, так и знания, относящиеся к данной конкретной ситуации.

Общностью апперцепционной базы объясняется большое количество неполных предложений в диалоге. Для диалогической речи характерен принцип экономии средств словесного выражения, проявляющийся не только в опущении заведомо известной информации, но также в обилии эллиптических предложений, когда наиболее предсказуемые и наименее информативные элементы предложения опускаются, делая речь более лаконичной, а темп диалога более быстрым. Как отмечает С.Г. Ильенко, в речевой практике неполные предложения могут оказываться более «полноценными», чем полные, поскольку сосредоточивают внимание слушателя на реме, отбрасывая уже известную слушателю тематическую часть [4, с. 47-48].

«В результате трансформации полного предложения в эллиптическое происходит усечение внешнего, формального состава предложения, однако семантический вес предложения трансформа нарастает, т.е. на исходную семантику структуры накладывается добавочная семантика» [5, с. 60]. Семантическая функция усечения предложения состоит в выделении ремы высказывания, при котором «тема высказывания («данное» в информации) сдвигается на положение общего фона, и рематическая часть высказывания («новое» в информации) становится единственным, уникальным элементом внешнего выражения» [2, с. 95]. Например:

Gaynor (straightening her dress): There. How's that look?

Humbolt: Splendid [12, p. 35].

Таким образом, в структуре оценочного эллиптического предложения остаётся лишь предикатив $\mathrm{P}\left(\mathrm{Adj}_{\mathrm{ev} / \mathrm{ex.ev}}\right)$. Смысл подобных эллиптических ответов в диалоге ясен и нет необходимости восстанавливать полную синтаксическую конструкцию. Кроме того, как отмечает Н.В. Ильина, «использование здесь полной конструкции исказило бы стилистический статус высказывания, поскольку краткие ответы на вопросы и на другие диалогические реплики являются нормой, а полные ответы - отклонением от этой нормы, возможным при

2 Ex.ev. - extreme evaluation / предельная оценка 
особых условиях с целью изменения стилистических оттенков» [5, с. 61].

Другим вариантом структуры эллиптического оценочного предложения может выступать $\mathrm{P}\left(\mathrm{Adj}_{\mathrm{ev} / \mathrm{ex} \text {.ev }}+\mathrm{N}\right)$. Например:

Phil.: I'm beginning to wonder if we're not all crackers.

Cud. (puzzled): Crackers?

Joe: She means cracked, daft, bug-house, barmy.

Cud.: Certainly not, Miss Loxfield. Silly idea! Don't feel at all cracked myself [18, p. 137];

Н.В.Ильина также выделяет оценочные предложения с элиминированным связочным глаголом [5, с. 65] S + P $\left(\operatorname{Adj}_{\text {ev.lex.ev }}+\mathrm{N}\right)$, типа: "You just stood by, didn't you? You stood by. You spineless little rat. I'm suing you for everything" [8, p. 69]. "You silly man. You silly woman. Handcuffing yourselves together. Don't do it. What for?" [9, p. 20];

Автор анализирует семантико-структурные особенности данной конструкции, имеющей спорный статус в работах исследователей, некоторые из которых относят её в разряд односоставных предложений, другие отождествляют с обращением или рассматривают как конструкции с аппозитивом. Н.В. Ильина относит их в разряд эллиптических оценочных конструкций на основании следующих доводов:

1. в данных предложениях утверждается оценка, относимая к объекту (субъекту предложения), выражаемая не только предикативным существительным, но и прилагательным (что противоречит понятию аппозитива);

2. подобные оценочные предложения имеют корреляцию с полносоставными предложениями;

3. в функции подлежащего, кроме личного местоимения уои, возможны и другие местоимения или имена существительные (что не позволяет им быть обращением) [5, с. 65-67].

Рассмотрим ещё пример: “He needn't think he's got away with it - 'cause he hasn't. He'll be a stretcher case tomorrow morning. (She screams up at the bedrooms.) You'll be a stretcher case tomorrow morning! You wait! You rotten yellow-bellied squint-eyed get! You're nothing else! You closet!" [19, p. 334]. Данный пример позволяет подтвердить статус анализируемых конструкций за счёт того, что эллиптические оценочные предложения You rotten yellow-bellied squint-eyed get!, You closet! употреблены в параллельной конструкции со структурно полным оценочным предложением You're nothing else!, что раскрывает их семантику и речевое намерение говорящего.

Другим способом экспрессивной модификации синтаксических конструкций в диалогической речи является включение в предложение уточняющего присоединения. Интенсивность оценки в этом случае увеличивается за счёт интенсификации предикации выдвинутым предикативом. В этом случае имеет место присоединение уточнения к эллиптическому предложению по следующей схеме $\mathrm{P}\left(\mathrm{Adj}_{\mathrm{ev} / \mathrm{ex.ev}}+\mathrm{N}\right)+\mathrm{S}$, например: "Nice little fella, Tommy, so long as you didn't have too much of him" [18, p. 138]; "Not very brainy, these Logans" [lbid: 139].

В таких случаях рема, собственно оценка, вынесена в начальную позицию и представлена отдельной интонационной единицей, что значительно утяжеляет коммуникативный вес предикатива и усиливает его семантическое содержание. При этом уточняющее присоединение содержит субъект предложения в нетипичной для него позиции и изолированно, что также интонационно выделяет его.

Таким образом, наряду с лексематическим уровнем создания интенсивности оценки (предельно-оценочные лексемы, лексические интенсификаторы степени), ситуативные нарушения исходной структуры оценочного предложения при построении оценочной диктемы, выраженные недостаточностью элементов, а также их инвертированным порядком, производят эффекта усиления, интенсификации отдельных частей или целой реплики.

\section{ЛИТЕРАТУРА}

1. Блох М.Я. Диктема в уровневой структуре языка // Вопросы языкознания. М.: Наука, 2000. - № 4. - С. 56-67.

2. Блох М.Я. Факультативные позиции и нулевые формы в парадигматическом синтаксисе // Проблемы синтаксиса английского языка. - М., 1970. - С. 75-106.

3. Блох М.Я., Кошман Ю.И. Категориальный статус предельной оценки // Филологические науки. Вопросы теории и практики. - Тамбов: Грамота, 2015. № 4 (46). Ч. 1. С. 38-40.

4. Ильенко С.Г. Русистика: Избранные труды. - СПб: Изд-во РГПУ, 2003. - 674 с.

5. Ильина Н.В. Структура и функционирование оценочных конструкций в современном английском языке: дис. ... канд. филол. наук: 10.02 .04$. - М., 1984. $-200 \mathrm{c}$.

6. Кошман Ю.И. Прагматическое содержание прямых и косвенных оценочных высказываний // Филологические науки. Вопросы теории и практики. Тамбов: Грамота, 2015. № 4 (46). Ч. 2. С.111-113.

7. Эпштейн, М.Н. Идеология и язык (Построение модели и осмысление дискурса) / М.Н. Эпштейн // Вопросы языкознания. - М.: Наука, 1991. - №6. - С. 19- 33.

8. Ayckbourn A. Way Upstream: A Play. - London: Samuel French, 1983. - 95 p.

9. Bennett A. Habeas Corpus: A Play in Two Acts. - London: Faber and Faber, 1973. - 75 p. 
10. Bradbury M. Love on a Gunboat // The After Dinner Game. - London: Arrow Books, 1982. - P. 79-132.

11. Bradbury M. Standing in for Henry // The After Dinner Game. - London: Arrow Books, 1982. - P. 133-176.

12. Bradbury M. The After Dinner Game // The After Dinner Game. - London: Arrow Books, 1982. - P. 21-78.

13. Longman Dictionary of Contemporary English Online [Электронный ресурс]. URL: www.Idoceonline.com/ (дата обращения: 20.03.21).

14. Macmillan Dictionary [Электронный ресурc]. URL: https://www.macmillandictionary.com/ (дата обращения: 20.03.21).

15. Osborne J. Look Back in Anger // Modern English Plays. - Moscow: Progress Publishing, 1966. - p. 27-142.

16. Pinero A.W. The Gay Lord Quex: A Comedy in Four Acts. - London: William Heinemann, 1903. - 225 p.

17. Priestley J.B. The Long Mirror // Four Plays. - London: Heinemann, 1944. - p. 59-117.

18. Priestley J.B. They Came to a City // Four Plays. - London: Heinemann, 1944. - p. 119-174.

19. Waterhouse K., Hall W. Billy Liar // Modern English Plays. - Moscow: Progress Publishing, 1966. - P. 233-335.

о Медведева Юлия Ивановна (Tea4english@yandex.ru).

Журнал «Современная наука: актуальные проблемы теории и практики»

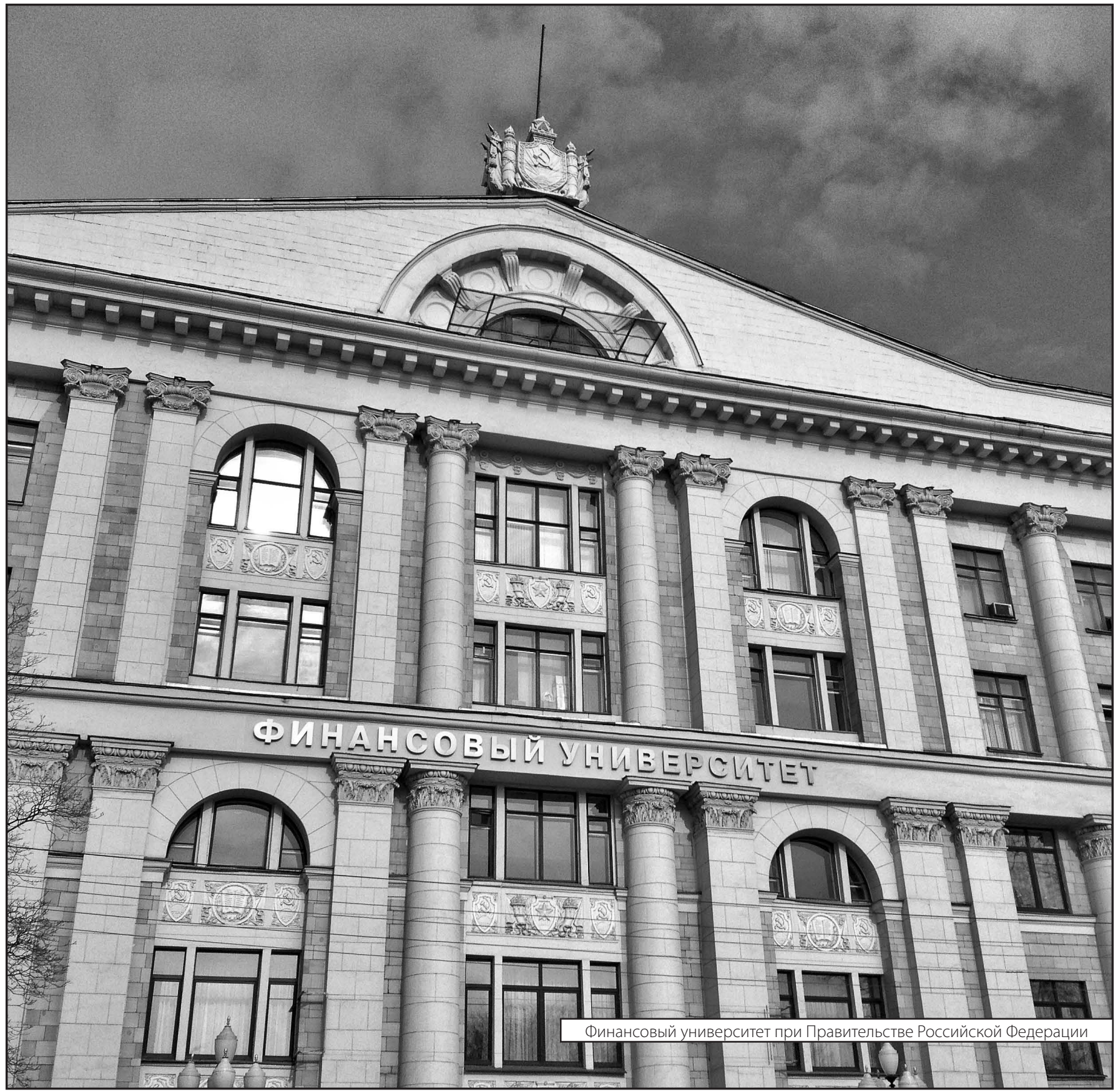

